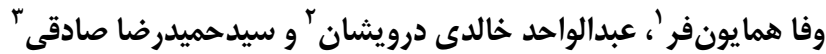

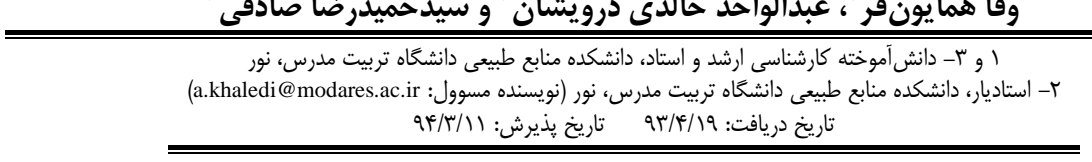

קكيده

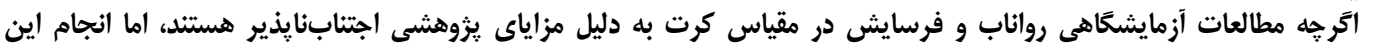

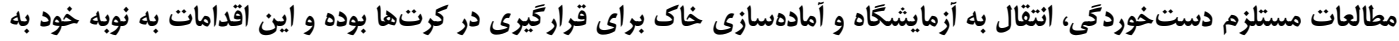

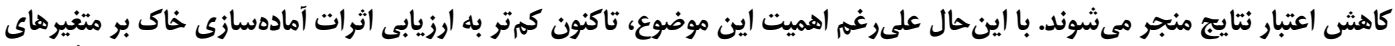

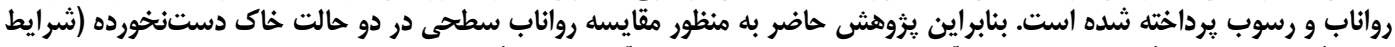

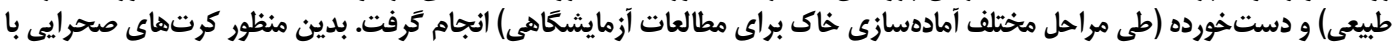

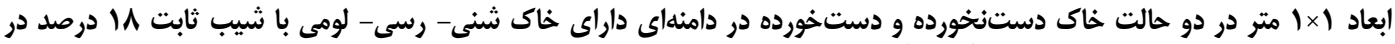

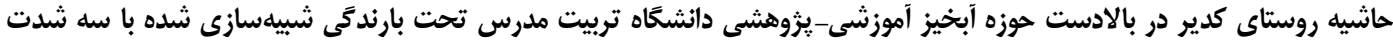

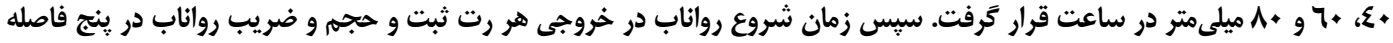

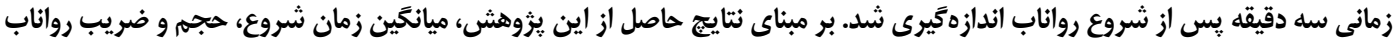

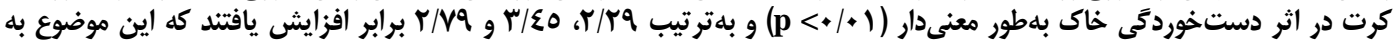

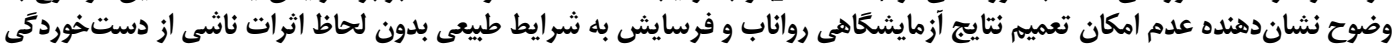

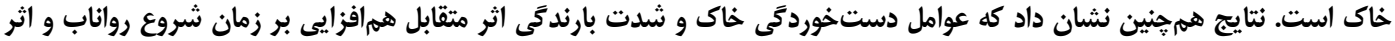
متقابل همزدايى بر حجم و ضريب روان رواناب داشتند.

وازههاى كليدى: زمان شروع رواناب، دستخوردَى خاك، شبيهساز باران، ضريب رواناب

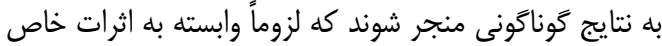

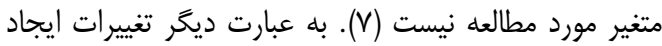

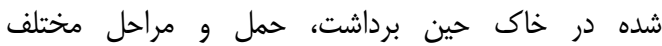

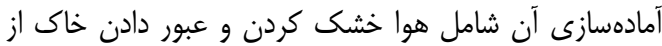

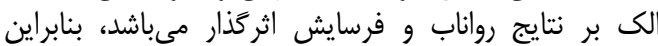

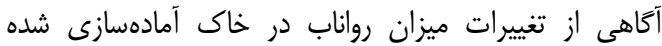

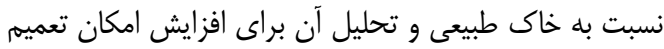

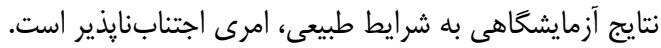

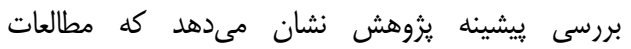

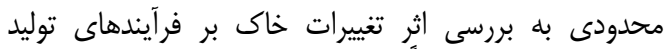

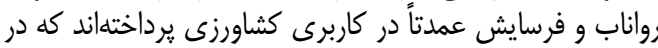

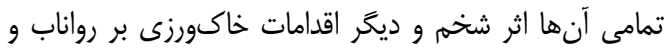

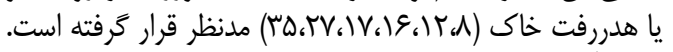

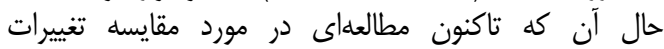

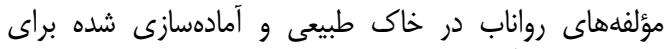

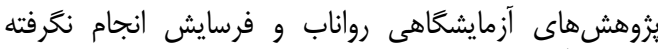

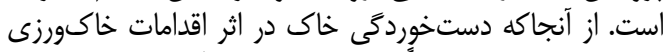

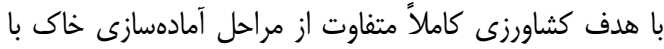

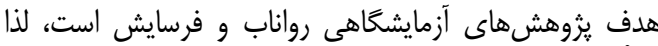

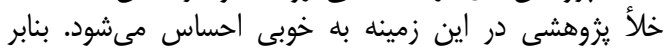

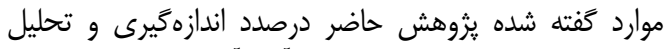

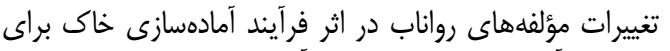

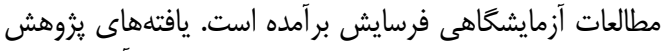

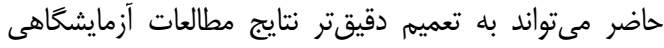
فرسايش خاك به شرايط طبيعى منجر شود.
مقدمه حلم

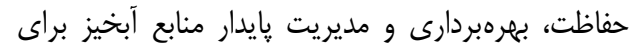

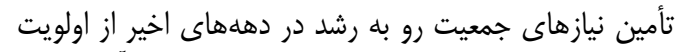

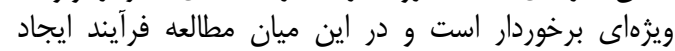

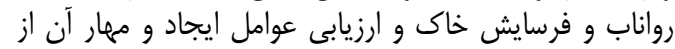

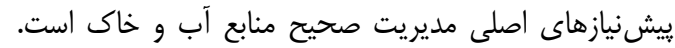

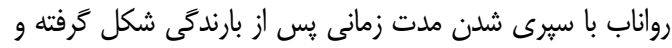

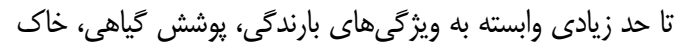

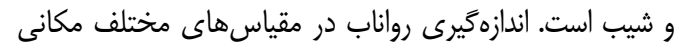

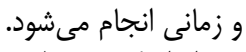

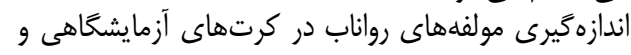

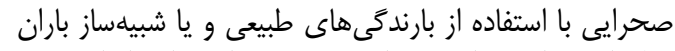

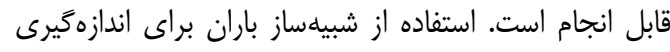

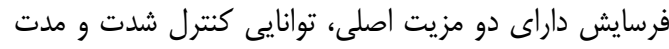

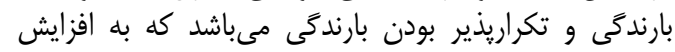

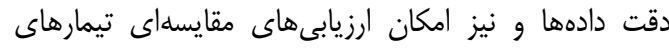

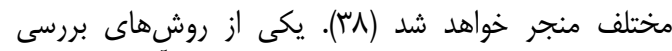

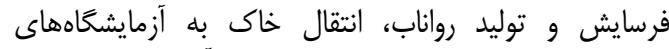

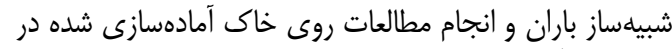
كرتهاى آزمايشكاهى است.

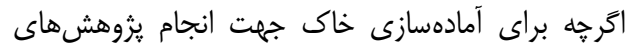

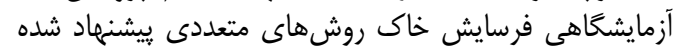

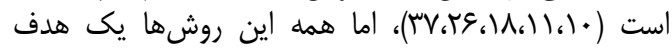

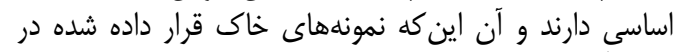

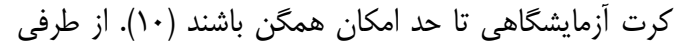

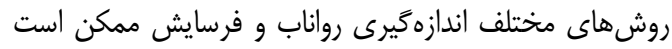


دامنه جنوبى مىباشد. موقعيت كلى محدوده اجراى يروهش

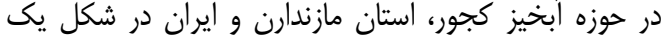

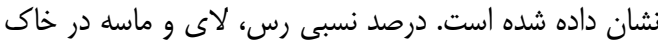

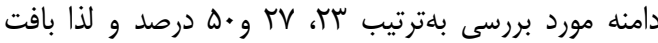

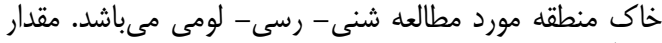
ماد آلى خاك من ماك

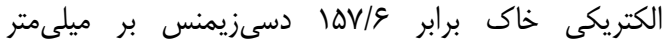

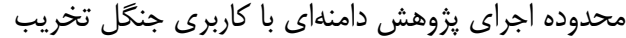

مواد و روشها

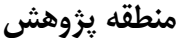
شده در حاشيه روستاى كدير و در شرق حوزه دائ آبخيز آموزشى و

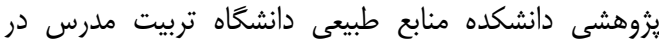

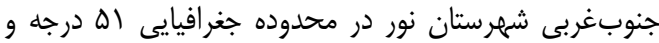

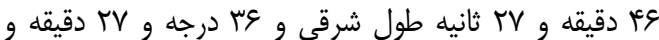

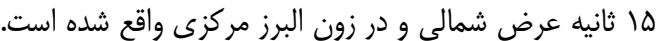

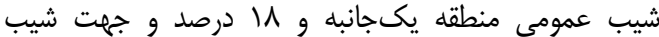

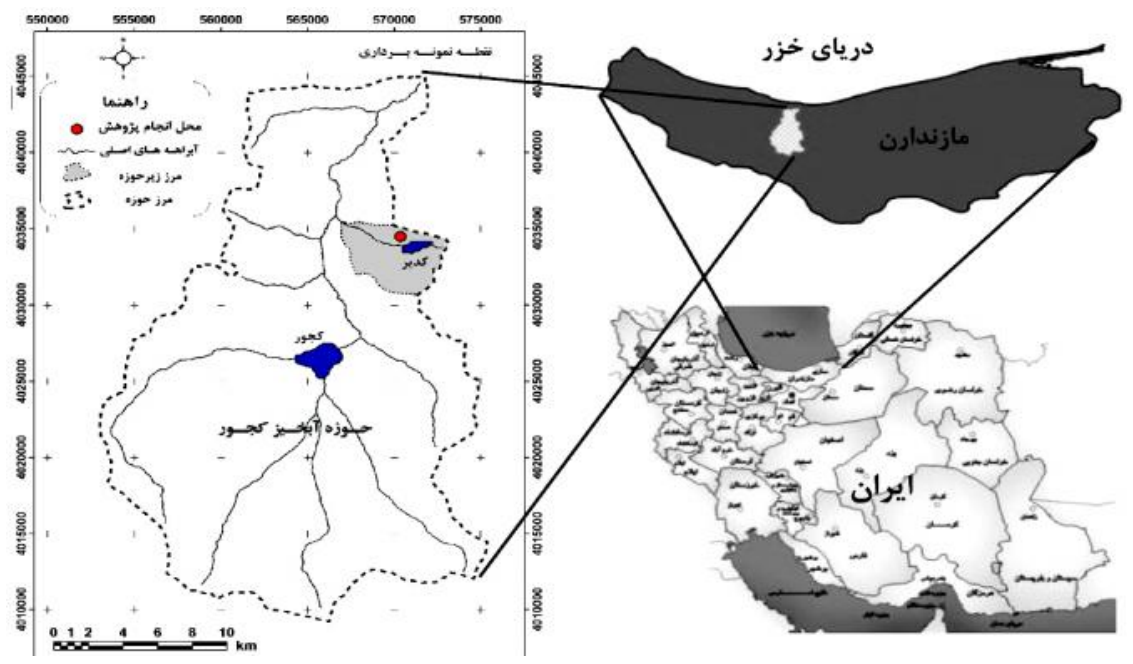

شكل ا- موقعيت دامنه مورد مطالعه در حوزه آبخيز كجور استان مازندران

Figure 1. The location of the study slope in Kojour watershed, Mazandaran province

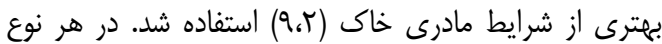

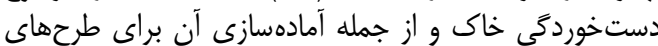

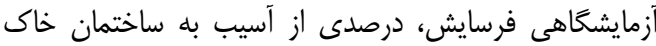

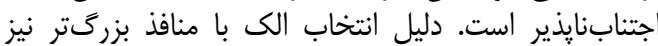

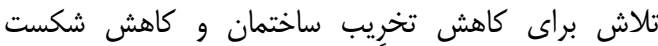

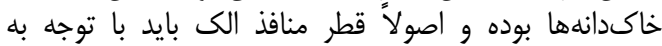

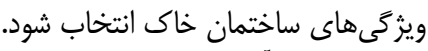

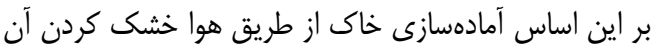

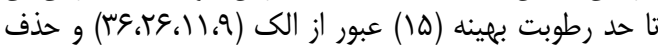

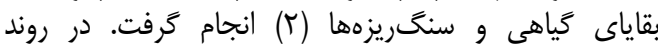

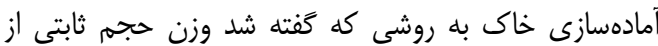

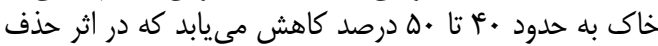

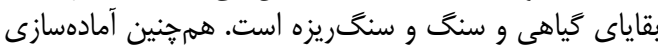

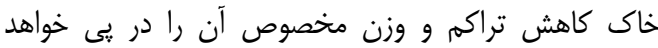
داشت. جدول ا مشخصات خاك قبل و و بعد از آمادهسازى را را

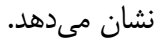

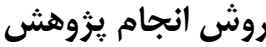

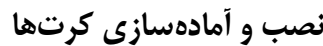

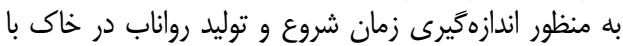

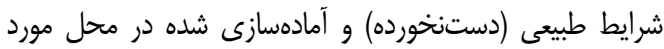

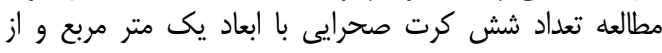

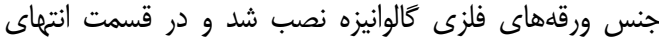

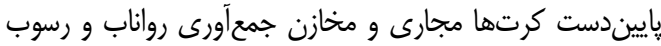

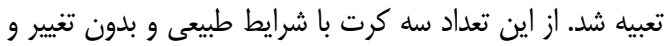

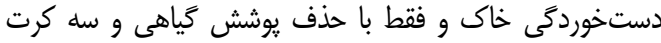

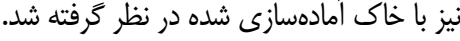

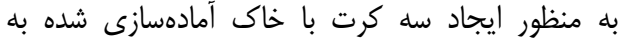

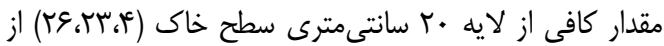

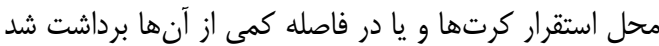

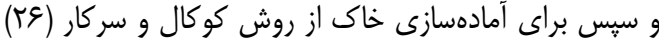

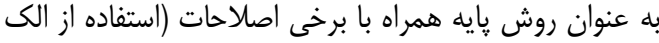

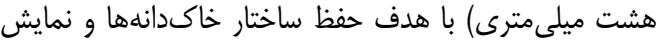

جدول ا- مشخصات خاك قبل و بعد از آمادهازى

Table 1. Soil characteristics before and after soil preparation

\begin{tabular}{|c|c|}
\hline ساختمان ظاهرى & (كرم بر سانتىمتر مكعب) \\
\hline دانهاى & $1 / 8$ \\
\hline دانهاى & $1 / \Delta$ \\
\hline
\end{tabular}




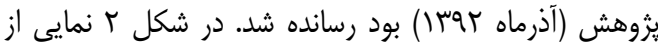

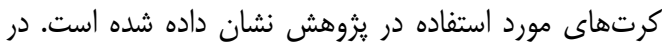

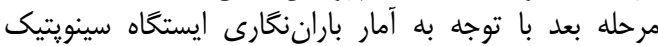

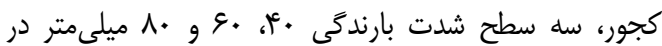

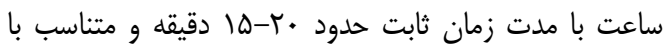

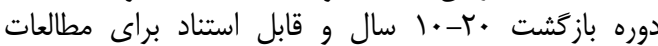

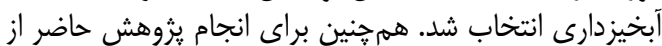

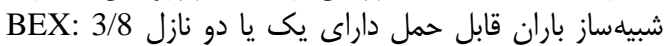

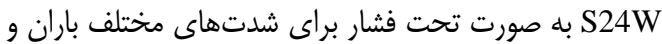
ارتفاع ريزش سه متر استفاده شد.
لذا يس از انتقال خاك به داخل كرتها با توجه به اهميت

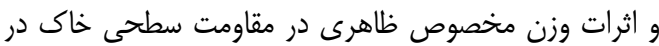

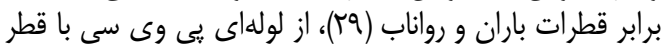

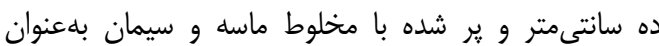

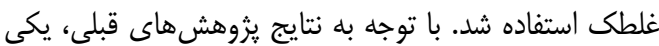

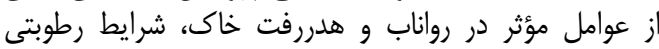

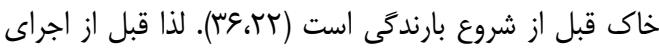

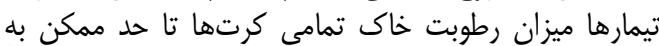

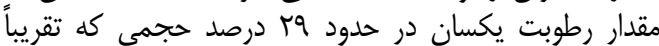
نشاندهنده شرايط هواخشى خاك در در فاصله زمانى انجام

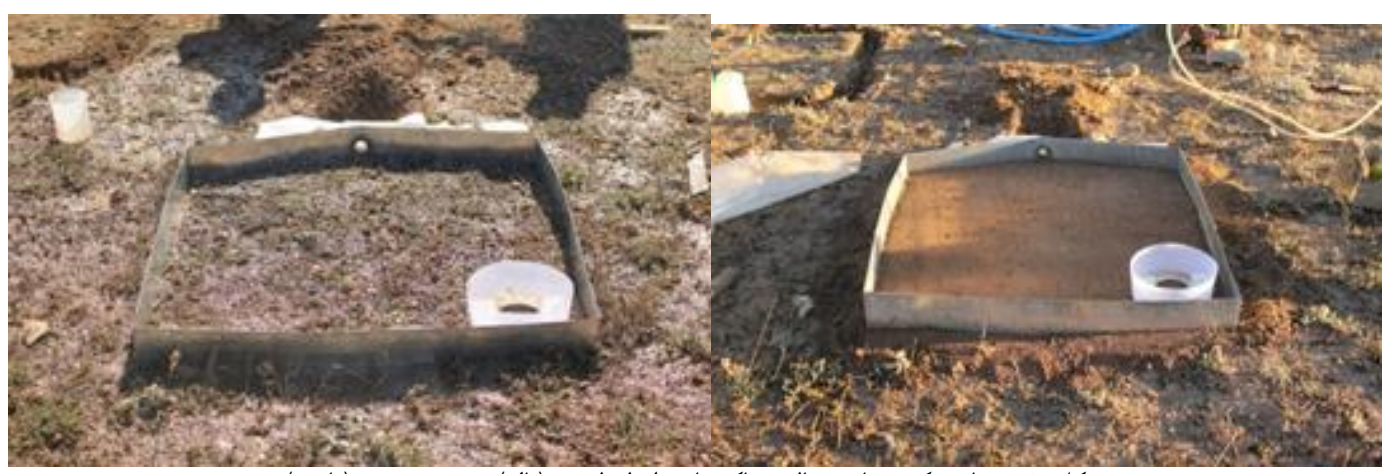

شكل r- تصاوير كرتها در حالت خاك با شرايط طبيعى (بالا) و دستخورده (يايين)

Figure 2. Views of the plots in natural soil conditions (up) and disturbed soil (down)

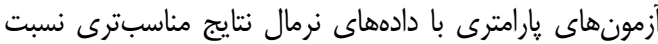

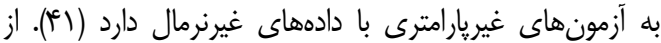

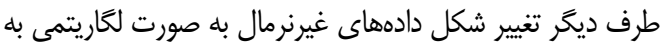

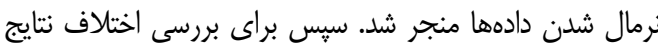

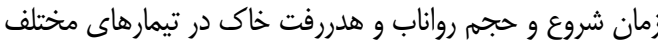

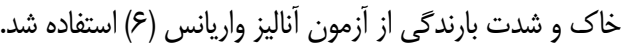

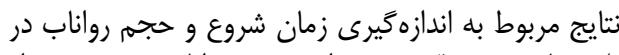

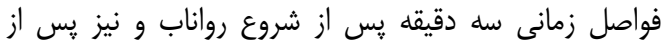

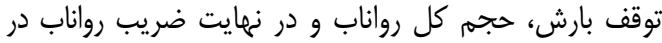

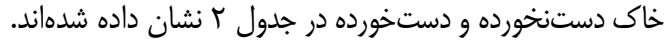

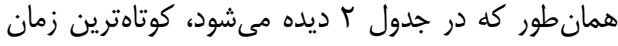

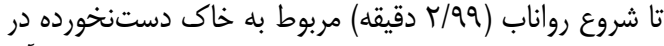

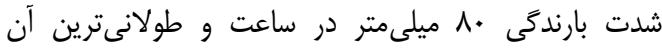

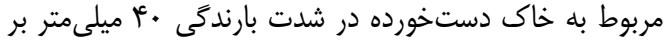

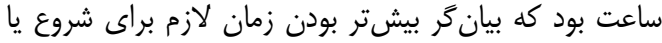

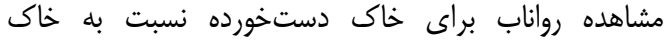

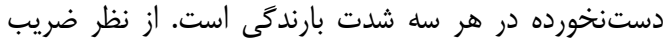

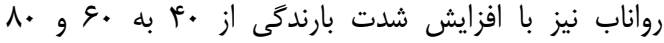

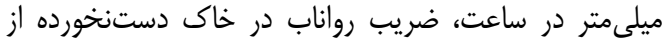

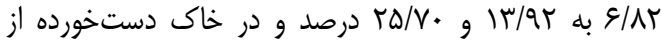

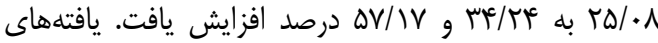
مذكور پِ از تحليل آمارى نتايج نيز تأييد شده و در جدول

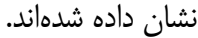

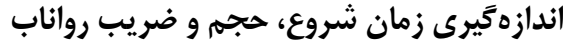
به منظور آكاهى از زمان زمان شروع و و تغييرات زمان زمانى رواناب

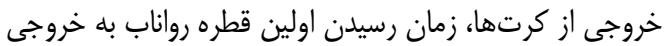

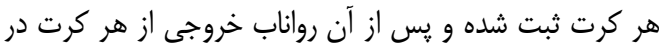

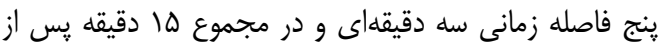

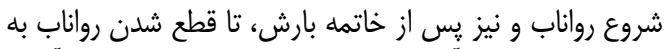

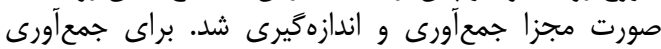

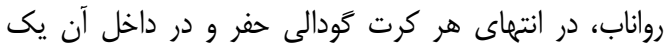

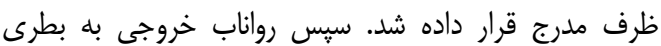

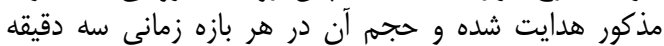

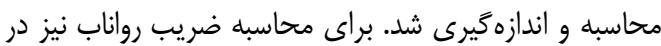

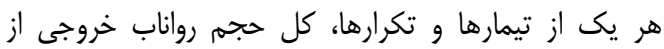

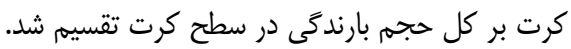

تحليل آمارى نتايج

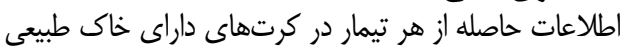

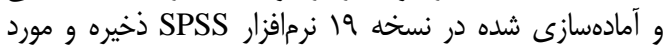

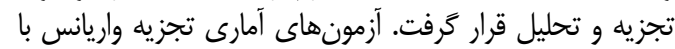

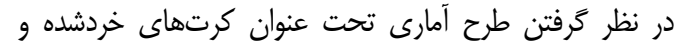

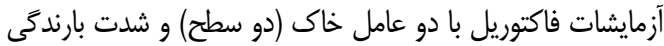

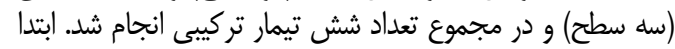

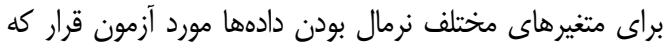

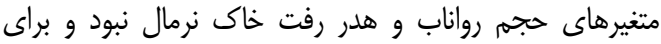

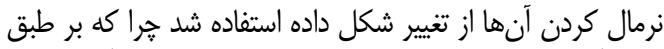

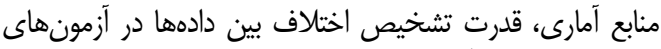

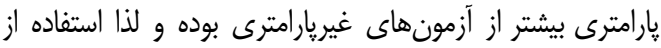



Table 2. The results of time to runoff, runoff volume and coefficient in three replications of studied treatments

\begin{tabular}{|c|c|c|c|c|c|c|c|c|c|c|}
\hline \multirow{3}{*}{ ضريب رواناب } & \multicolumn{7}{|c|}{ حجم رواناب (ليتر) } & \multirow{3}{*}{ زرواناب (دقيقه) } & \multirow{3}{*}{ 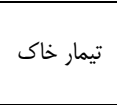 } & \multirow{3}{*}{ شيلى متر در ساعتى } \\
\hline & حجم كل & يس از توقف & \multicolumn{5}{|c|}{ در زمان حس از شروع رواناب (دقيقه) } & & & \\
\hline & رواناب & بارش & 10 & ir & 9 & 4 & r & & & \\
\hline g/AT & $V / \cdot \Delta V$ & $\cdot / \cdot 0$ & .1199 &.$/ I V T$ &.$/ 1 F \mathrm{~V}$ & . & .1 .91 & N/DF & دستنخورده & \\
\hline$r \Delta / \cdot \Lambda$ & $f / r q 1$ & $.|| Q \mid$ &.$/ 94 \wedge$ &.$/ N \& 8$ &.$/ V 11$ &.$/ 499$ &.$/$ the & عس/11 & دستخورده & \\
\hline $1 \% / 94$ & $r / 9 \mid \Lambda$ &.$/ 1 T \Lambda$ & $\cdot / V T A$ & . &.$/ \Delta 1 Q$ & $\cdot / f I f$ &.$/ 4 I T$ & ए/११ & دستنخورده & \\
\hline$M F / T F$ & $1 . / 1 \mathrm{~V}$ & ./TFD & r/ADT & r/VTr & T/IT. & $1 / \Delta \cdot \Lambda$ & .1990 & $\mid Q / V T^{f}$ & دستخورده & \\
\hline$r \omega / v \cdot$ & $8 / T \cdot 1$ &.$/ 411$ & I/TIE & I/KI &.$/ 910$ & . /VGa &.$/ T \omega$. & $r / 99$ & دستنخورده & А. \\
\hline$\Delta V / \mathrm{VV}$ & $\mid f / q$. &.$/ 791$ & $r / R$. & $r / \Delta V q$ & $r / 9 \mid \Lambda$ & $T / \Lambda \cdot \Delta$ & ./19८ & $r / V r$ & دستخورده & \\
\hline
\end{tabular}

شروع رواناب كاهش يافت كه ضمن وجود انتظار اين فرآيند

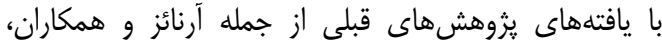

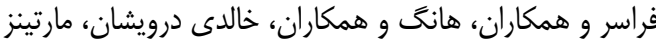

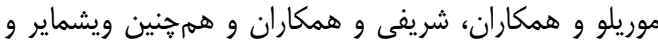

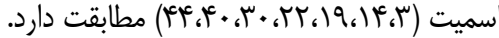

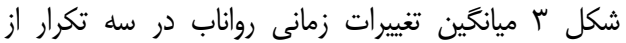

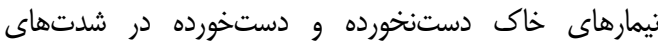

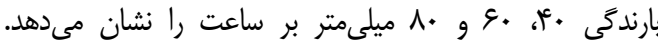

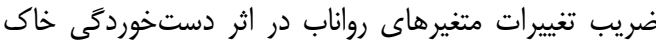

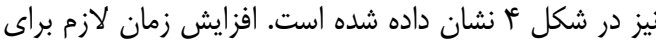

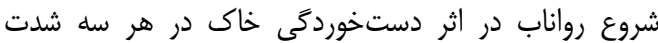

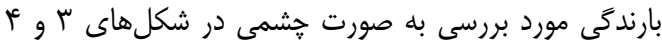
نيز قابل تشخيص است مورد برسي

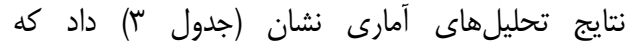

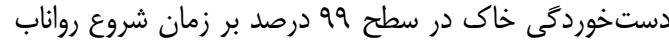

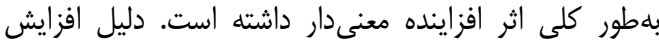

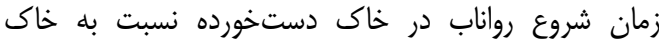

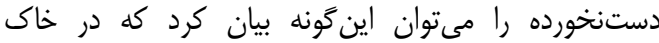

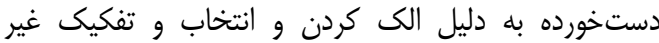

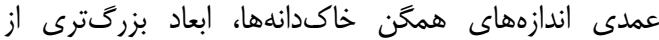

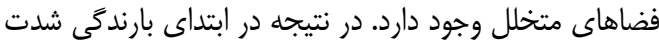

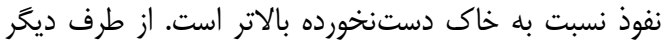

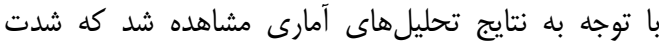

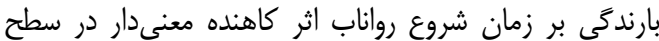

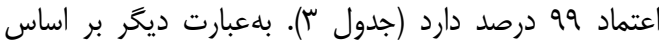

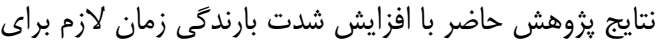

جدول ץ- اثرات شدت بارندگى و دستخوردگى خاك بر زمان شروع، حجم و ضريب رواناب Table 3. The effects of rainfall intensity and soil disturbance on time to runoff, runoff volume and coefficient

\begin{tabular}{|c|c|c|c|c|c|c|}
\hline سطح معنى دارى & Fقدار F F F & ميانگين مربعات & درجه آزادى & مجموع مربعات & متغيير وابسته & منبع \\
\hline$\cdot / \ldots{ }^{4}$ & 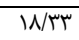 & $V \pi / T$ & & $1 f e / 4 \mathrm{~V}$ & زمان شروع رواناب (دقيقه) & \\
\hline$\cdot / \cdot r^{*}$ & $9 / 9 \Lambda$ & $\cdot / V T$ & r & $1 / \& \Delta$ & لكاريتم حجم رواناب (ليتر) & شدت بارندگى \\
\hline$\cdot / \cdot 1 \mu^{*}$ & س & $1.18 / \wedge 8$ & & $r \cdot r \mu / q$. & ضريب رواناب (درصد) & \\
\hline$\cdot / \ldots{ }^{*}$ & r & 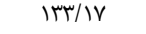 & & I IT/IV & زمان شروع رواناب (دقيقه) & \\
\hline$\cdot / .+1^{\text {wats }}$ & $r \cdot / r \Lambda$ & I/AT & 1 & I/DT & لغاريته حجم رواناب (ليتر) & ت تيمار خاك \\
\hline$\cdot / . . r^{*}$ & $\mid Q / \Delta Y$ & TEAT/T. & & TEAT/T. & ضريب رواناب (درصد) & \\
\hline
\end{tabular}

افزايش يافته است. بلنظر مىرسد با افزايش شدت بارت بارندكى اثر

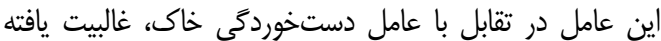

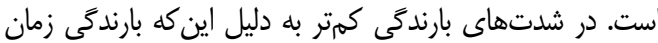

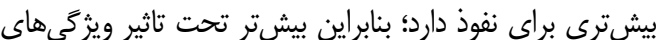

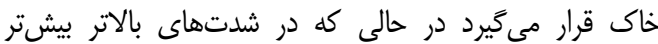

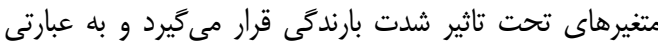

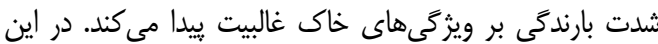

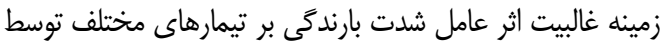

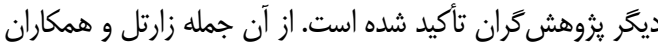

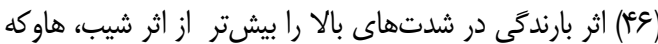

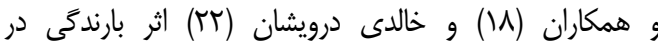

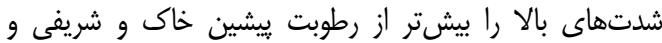

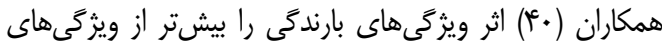
خاك دانستهاند.

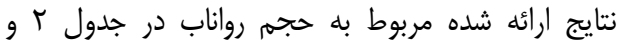

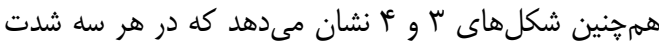

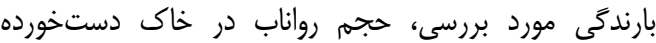

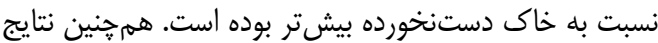

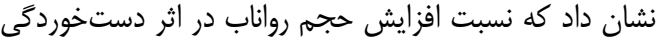

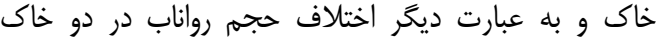

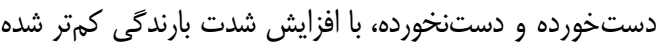

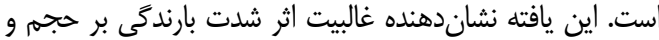

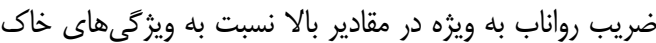

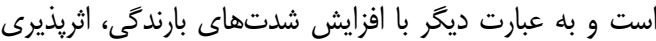

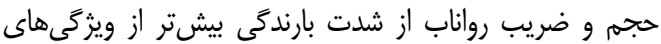
خاك است.

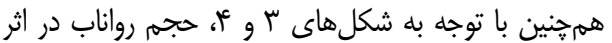

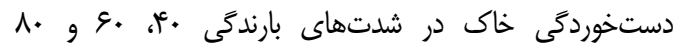

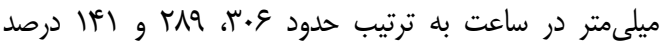



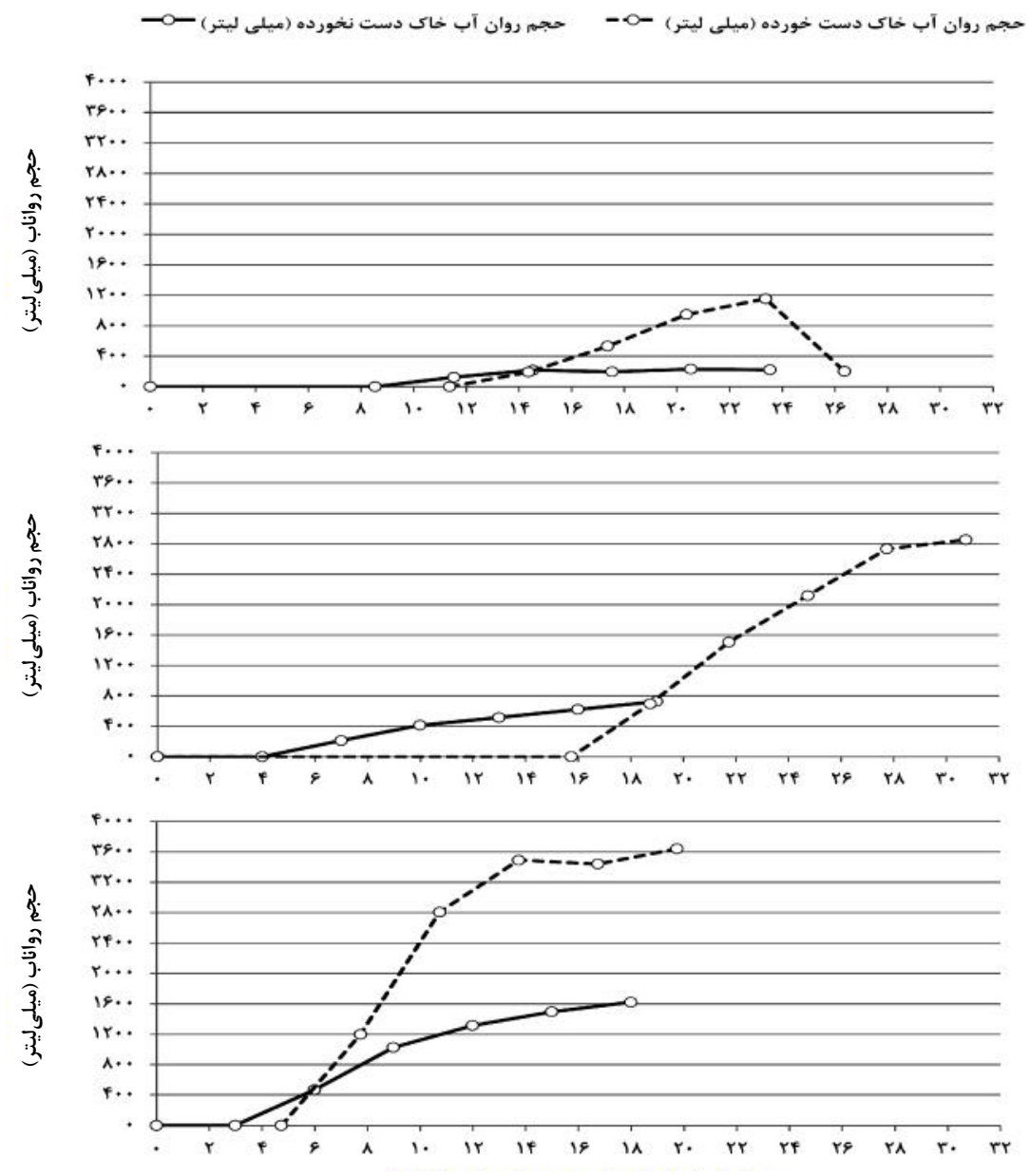

زمان انجام آزمايش از شروع بارندكى (دقيقه)

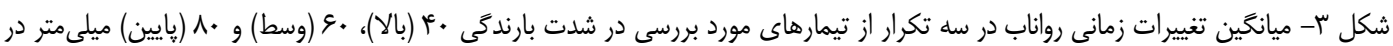
ساعت

Figure 3. The average temporal variations of runoff in three replications of studied treatments in rainfall intensities of 40 (up), 60 (middle) and 80 (down) $\mathrm{mm} \mathrm{h}^{-1}$

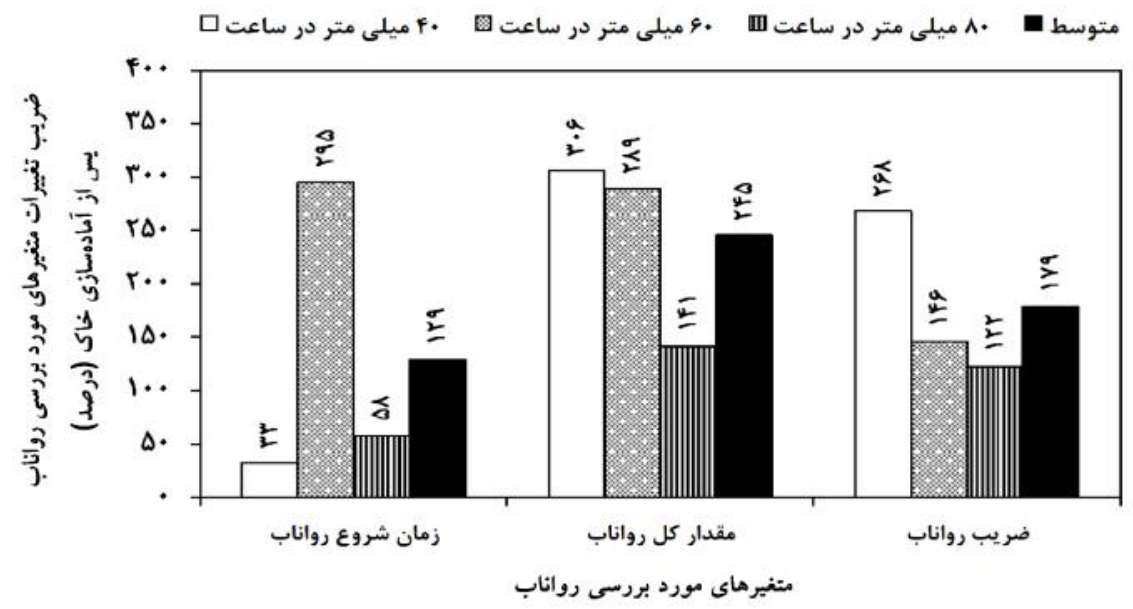

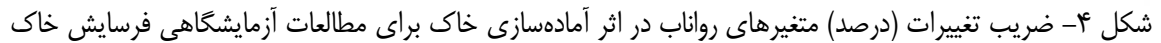

Figure 4. Variation coefficient (\%) of runoff variables due to the soil preparation for laboratory soil erosion studies 
همرجنين با توجه به جدول يك ضريب رواناب در هر هر سه

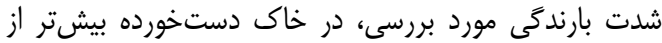

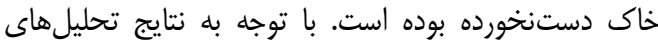

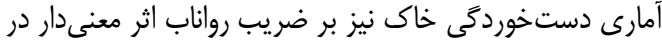

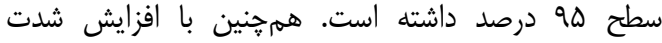

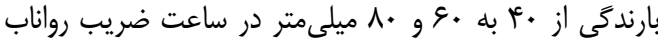

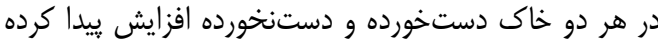

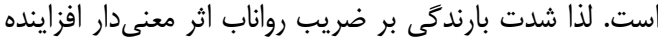

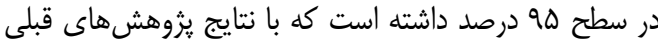

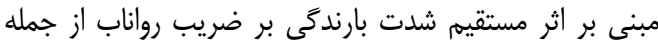

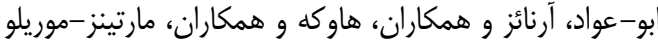

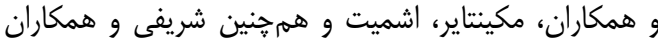

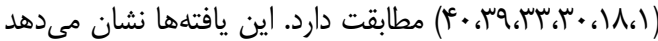

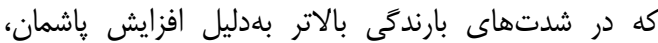

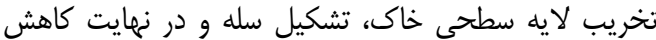

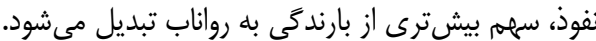

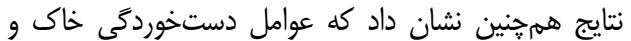

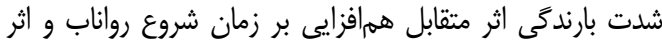

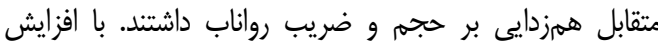

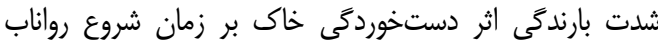

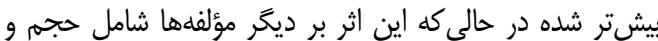

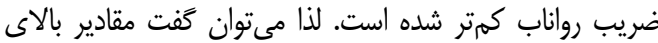

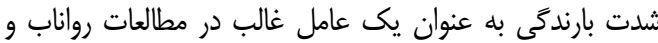

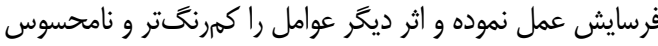

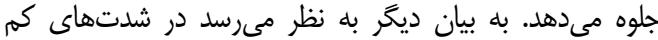

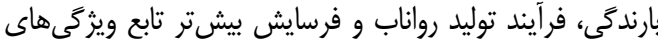

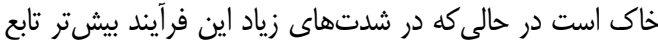

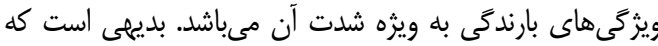

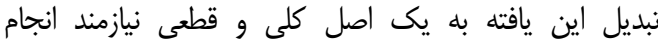

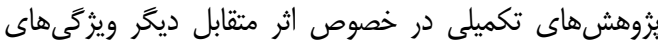

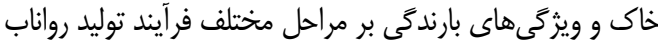

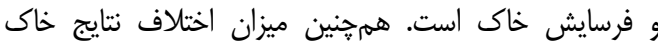

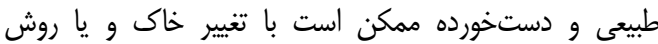

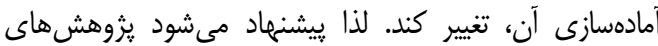

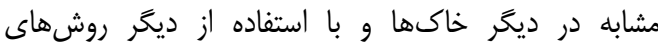

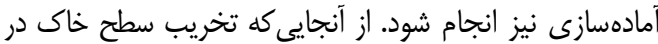

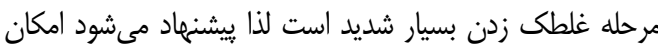

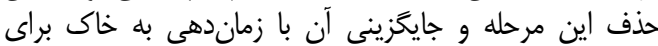
بازيابى ساختار نسبى نيز مورد بررسى جرئ قرار كَيرد.

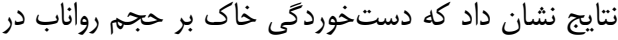

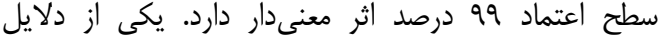

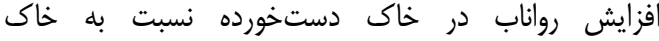

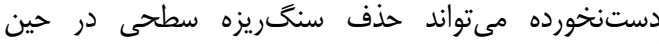

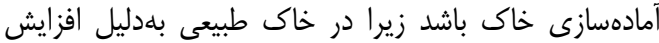

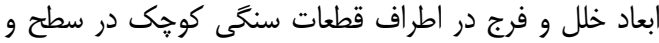

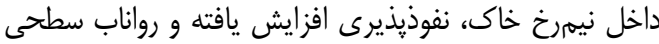

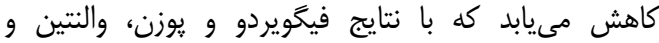

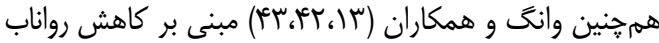
با افزايش درصد سنگ

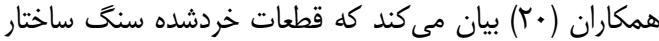

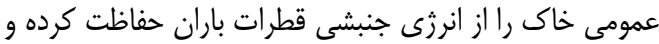

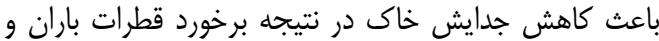

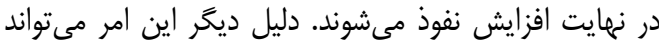

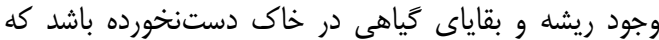

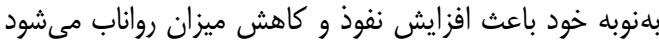

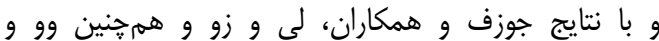

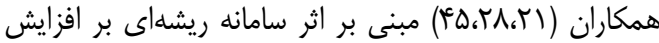

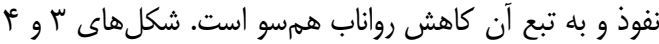

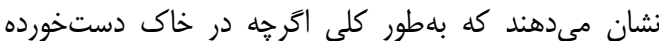

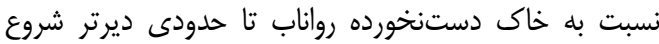

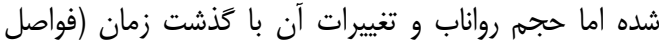

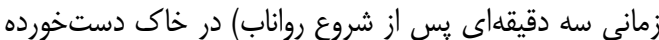

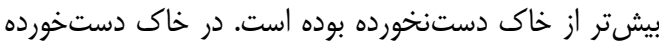

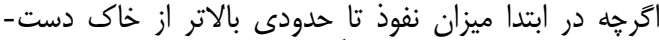

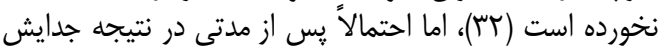

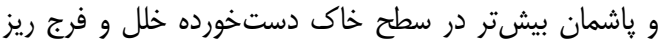

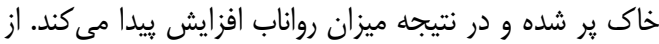

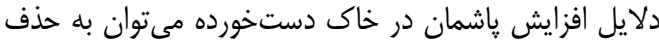

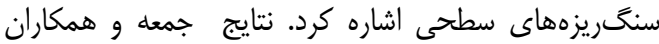

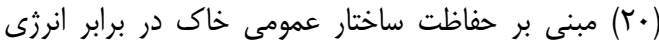

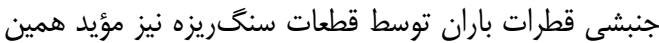

نكته مي باشد.

با توجه به نتايج تحليلهاى آمارى مشاهده شد كه شدات

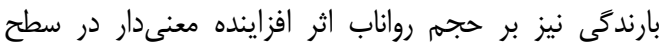

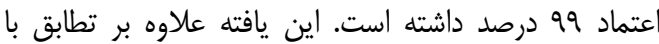

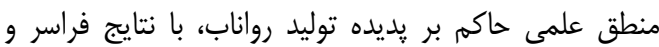

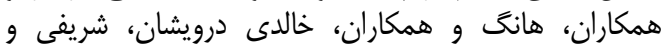

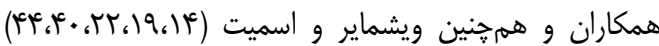

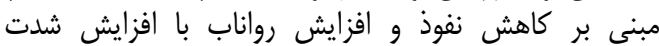
بارندگى همسو است. 
. Abu-Awwad, A.M. 1997. Water Infiltration and Redistribution within Soils Affected by a Surface Crust. Journal of Arid Environments, 37: 231-242.

2. Agassi, M. and J.M. Bradford. 1999. Methodologies for Interrill Soil Erosion Studies. Soil and Tillage Research, 49: 277-287.

3. Arnaez, J. T. Lasanta, P. Ruiz-Flano and L. Ortigosa. 2007. Factors Affecting Runoff and Erosion under Simulated Rainfall in Mediterranean Vineyards. Soil and Tillage Research, 93: 324-334.

4. Assouline, S. and M. Ben-Hur. 2006. Effects of Rainfall Intensity and Slope Gradient on the Dynamics of Interrill Erosion during Soil Surface Sealing. Catena, 66: 211-220.

5. Barthes, B. and E. Roose. 2002. Aggregate Stability as an Indicator of Soil Susceptibility to Runoff and Erosion; Validation at Several Levels. Catena, 47: 133-149.

6. Bihamta, M.R. and M.A. Zare Chahoki. 2011. Statistics Principles in Natural Resources, $2^{\text {nd }}$ Edn., Tehran University Press, Iran. 300 pp (In Persian).

7. Boardman, J., J.A. Dearing and I.D.L. Foster. 1990. Soil Erosion Studies, Some Assessments. In Soil Erosion on Agricultural Land. J. Boardman, I.D.L. Foster and J.A. Dearing (eds.), Wiley, New York. 1990: 659-672.

8. Choudhary, M.A., A.R. Lal and W.A. Dick. 1997. Long-term Tillage Effects on Runoff and Soil Erosion under Simulated Rainfall for a Central Ohio Soil. Soil and Tillage Research, 42: 175-184.

9. Defersha, M.B., S. Quraishi and A.M. Mellese. 2011. The Effect of Slope Steepness and Antecedent Moisture Content on Interrill Erosion, Runoff and Sediment Size Distribution in the Highlands of Ethiopia. Hydrology and Earth System Sciences, 15: 2367-2375.

10. Ekwue, E.I. 1991. The Effects of Soil Organic Matter Content, Rainfall Duration and Aggregate Size on Soil Detachment. Soil Technology, 4: 197-207.

11. Ekwue, E.I. and A. Harrilal. 2010. Effect of Soil Type, Peat, Slope, Compaction Effort and Their Interactions on Infiltration, Runoff and Raindrop Erosion of Some Trinidadian Soils. Biosystems Engineering, 105: 112-118.

12. Erkossa, T., K. Stahr and T. Gaiser. 2005. Effect of Different Methods of Land Preparation on Runoff, Soil and Nutrient Losses from a Vertisol in the Ethiopian Highlands, Soil Use and Management, 21: 253-259.

13. Figueiredo, T. and J. Poesen. 1998. Effects of Surface Rock Fragment Characteristics on Interrill Runoff and Erosion of a Silty Loam soil. Soil and Tillage Research, 46: 81-95.

14. Fraser, I., T.R. Harrod and P.M. Haygarth. 1999. The Effect of Rainfall Intensity on Soil Erosion and Particulate Phosphorus Transfer from Arable Soils. Water Science and Technology, 39: 41-45.

15. Fox, D.M. and R.B. Bryan. 1999. The Relationship of Soil loss by Interrill Erosion to Slope Gradient. Catena, 38: 211-222.

16. Gomez, J.A. and M.A. Nearing. 2005. Runoff and Sediment Losses from Rough and Smooth Soil Surfaces in a Laboratory Experiment. Catena, 59: 253-266.

17. Harold, L.B., A.G. Mccall and F.G. Bell. 1945. Investigations in Erosion Control and Reclamation of Eroded Land at the Northwest Appalachian Conservation Experiment Station, Zanesville, Ohio, 193442. United States Department of Agriculture, Technical Bulettin 888, 95 pp.

18. Hawke, R.M., A.G. Price and R.B. Bryan. 2006. The effect of initial soil water content and rainfall intensity on near-surface soil hydrologic conductivity, a laboratory investigation. Catena, 65: 237-246.

19. Huang, J., P.T. Wu and X.N. Zhao. 2010. Impact of Slope Biological Regulated Measures on Soil Water Infiltration. Transactions of the CSAE, 26: 29-37.

20. Jomaa S., D.A. Barry, A. Brovelli, B.C.P. Heng, G.C. Sander, J.Y. Parlange and C.W. Rose. 2012. Rain Splash Soil Erosion Estimation in the Presence of Rock Fragments. Catena, 92: 38-48.

21. Joseph, L., J. Pikul and J.A. Kristian. 2003. Water Infiltration and Storage Affected by Subsoiling and Subsequent Tillage. Soil Science Society of America Journal, 67: 859-867.

22. Khaledi Darvishan, A. 2013. Simulation of Runoff Generation and Soil Erosion Processes in Different Antecedent Soil Moisture Contents. Ph.D. Thesis, Department of Watershed Management Engineering, Faculty of Natural Resources, Tarbiat Modares University. 138 pp (In Persian).

23. Khaledi Darvishan, A., S.H.R. Sadeghi, M. Homaee and M. Arabkhedri. 2012. Potential Use of Synthetic Color-Contrast Aggregates and a Digital Image Processing Technique in Soil Splash Measurements. In Erosion and Sediment Yields in the Changing Environment, IAHS Publication 356: Wallingford, Oxfordshire, UK, 2012: 364-368.

24. Khaledi Darvishan, A., S.H.R. Sadeghi, M. Homaee and M. Arabkhdri. 2014. Measuring Sheet Erosion Using Synthetic Color-Contrast Aggregates. Hydrological Processes, 25: 4463-4471.

25. Kukal, S.S. and M. Sarkar. 2010. Splash Erosion and Infiltration in Relation to Mulching and Polyviny1 Alcohol Application in Semi-Arid Tropics. Archives of Agronomy and Soil Science, 56: 697-705.

26. Kukal, S.S. and M. Srakar. 2011. Laboratory Simulation Studies on Splash Erosion and Crusting in Relation to Surface Roughness and Raindrop Size. Journal of the Indian Society of Soil Sciences, 59: 87-93. 
27. Layon, T.L., H.O. Buckman and N.C. Brady. 1952. The Nature and Properties of Soil. 12th ed., Mac Millan Co., New York, 591 pp.

28. Li, Y. and X.Q. Xu. 1992. The Intensifying Effect of Plant Roots on the Soil Antiscouribility on the Loess Plateau. Science China, Series B, 3: 254-259.

29. Luk, S.H. 1985. Effect of Antecedent Soil Moisture Content on Rainwash Erosion. Catena, 12: 129139.

30. Martínez-Murillo, J.F., E. Nadal-Romero, D. Regüés, A. Cerdà and J. Poesen. 2013. Soil Erosion and Hydrology of the Western Mediterranean Badlands throughout Rainfall Simulation Experiments: A Review. Catena, 106: 101-112.

31. Martinez-Zavala, L., A. Jordan Lopez and N. Bellinfante. 2008. Seasonal Variability of Runoff and Soil Loss on Forest Road Backslopes under Simulated Rainfall. Catena, 74: 73-79.

32. Mazaheri, M.R., M. Mahmudabadi and Z. Rashidi. 2012. Infiltration Comparison at Two Natural and Laboratory Conditions with Emphasis on Soil Disturbance. $11^{\text {th }}$ National Seminar on Irrigation and Evapotranspiration, Shahid Bahonar University, Kerman, Iran. 7-10 January 2012, 9 pp (In Persian).

33. Mcintyre, D.S. 1958. Permeability Measurements of Soil Crusts Formed by Raindrop Impact. Soil Science, 85: 85-189.

34. Morgan, R.P.C. 2005. Soil Erosion and Conservation, Blackwell, Oxford, 316 pp.

35. Nikkami, D., A.J. Ardakani, F.B. Movahedi and P. Razmjoo. 2004. The Effects of Plough on Surface Runoff. FAO, $7 \mathrm{pp}$.

36. Orsham, A., A.M. Akhund Ali and A. Behnia. 2010. Effect of Soil Antecedent Moisture Contents on Runoff and Sedimentation Values with Simulated Rainfall Method. Iranian Journal of Range and Desert Reseach, 16: 445-455 (In Persian).

37. Romkens, M.J.M., K. Helming and S.N. Prasad. 2001. Soil Erosion under Different Rainfall Intensities, Surface Roughness and Soil Water Regimes. Catena, 46: 103-123.

38. Sadeghi, S.H.R. 2011. Study and Measurement of Water Erosion. Tarbiat Modares University Press, 200 pp (In Persian).

39. Schmidt, J. 2006. Effects of Soil Slaking and Sealing on Infiltration-Experiments and Model Approach. In: Proceedings of the $18^{\text {th }}$ World Congress of Soil Science, Philadelphia, Pennsylvania, USA, 2006: 29-32.

40. Sharifi, F.A.S., Sh. Safarpour, S.A. Ayoubzadeh and J. Vakilpour. 2005. An Investigation of Factors Affecting Runoff Generation in Arid and Semi-Arid Area Using Simulation and Rainfall Runoff Data. Iranian Journal of Natural Resources, 57: 33-47 (In Persian).

41. Townend, J. 2002. Practical Statistics for Environmental and Biological scientists, Chichester: John Wiley and Sons, $286 \mathrm{pp}$.

42. Valentin, C. 1994. Surface Sealing as Affected by Various Rock Fragment Covers in West Africa. Catena, 23: 87-97.

43. Wang, X., Z. Li, C. Cai, Z. Shi, Q. Xu, Z. Fu and Z. Guo. 2012. Effects of Rock Fragment Cover on Hydrological Response and Soil Loss from Regosols in a Semihumid Environment in South-West China. Geomorphology, 151-152: 234-242.

44. Wischmeier, W.H. and D.D. Smith. 1978. Predicting Rainfall Erosion Losses: A Guide to Conservation Planning. Agricultural Handbook No. 537. United States Department of Agriculture, Washington, DC, $58 \mathrm{pp}$.

45. Wu, W.D., S.Z. Zheng and Z.H. Lu. 2000. Effect of Plant Roots on Penetrability and Anti-Scouribility of Red Soil Derived from Granite. Pedosphere, 10: 183-188.

46. Zartl, A.S., A. Klik and C. Huang. 2001. Soil Detachment and Transport Processes from Interrill and Rill Areas. Physics and Chemistry of the Earth (B), 26: 25-26. 


\title{
Effects of Soil Preparation for Laboratorial Erosion Studies on Surface Runoff
}

\author{
Vafa Homauonfar ${ }^{1}$, Abdolvahed Khaledi Darvishan ${ }^{2}$ and Seyyed Hamid Reza Sadeghi ${ }^{3}$ \\ 1 and 3- M.Sc. Student and Professor, Faculty of Natural Resources, Tarbiat Modares University \\ 2- Assistant Professor, Faculty of Natural Resources, Tarbiat Modares University \\ (Corresponding author: a.khaledi@modares.ac.ir) \\ Received: July 10, $2014 \quad$ Accepted: June 1, 2015
}

\begin{abstract}
Despite of soil disturbance, transfer to the laboratory and preparation inside the plots which themselves lead to lower validity of the results, the experimental study on runoff and erosion at plot scale with many research advantages are inevitable. However, there has been less attention to evaluate the effects of soil preparation on runoff and sediment variables. The present study was therefore conducted to compare surface runoff in two undisturbed (natural) and disturbed (during various steps of preparing soil for laboratorial studies) soil conditions. To achieve the study purposes, $1 \times 1 \mathrm{~m}$-plots were considered in a slope with sandy-clay-loam soil and gradient of $18 \%$ under simulated rainfall with three intensities of 40,60 and $80 \mathrm{~mm} \mathrm{~h}^{-1}$. The study slope was located in the vicinity of Kodir village in upper Educational and Research Forest Watershed of Tarbiat Modares University. The time-to-runoff in the output of each plot was recorded and the runoff volume and coefficient at five three-minute intervals was subsequently measured. The results showed that the average time-to-runoff, runoff volume and coefficient were significantly ( $\mathrm{s} \triangle_{0.01}$ ) increased $2.29,3.45$ and 2.79 times, respectively due to the soil disturbance which clearly indicated the impossibility generalization of laboratorial runoff and erosion results to natural conditions without considering the effects of soil disturbance. The results showed the synergistic interaction of soil disturbance and rainfall intensity on time-torunoff and antagonistic interaction on runoff volume and coefficient.
\end{abstract}

Keywords: Rainfall Simulator, Runoff Coefficient, Soil Disturbance, Time-to-Runoff 